\title{
Fetal Ultrasound as a Window into Maternal Health
}

\author{
${ }^{1}$ Kelly Yamasato, ${ }^{2}$ Janet Burlingame
}

\begin{abstract}
Healthcare providers commonly use obstetric ultrasound to assess fetal well-being. However, the potential for fetal findings to manifest maternal disease is often underappreciated. This article will review the relationships between obstetric ultrasound findings and underlying maternal medical conditions in three settings: (1) fetal growth abnormalities related to underlying maternal disease, (2) congenital malformations secondary to diabetes and maternal exposure to chemical and infectious teratogens, and (3) congenital heart block due to maternal autoimmune disease. The obstetric ultrasound should be a tool used for the care and evaluation of the maternal as well as the fetal patient.
\end{abstract}

Keywords: Autoimmune diseases, Congenital heart block, Diabetes mellitus, Fetal alcohol syndrome, Fetal macrosomia, Intrauterine growth retardation, Maternal exposure, Teratogen, Ultrasound prenatal.

How to cite this article: Yamasato K, Burlingame J. Fetal Ultrasound as a Window into Maternal Health. Donald School J Ultrasound Obstet Gynecol 2016;10(1):37-41.

Source of support: Nil

Conflict of interest: None

\section{INTRODUCTION}

Ultrasound is a useful tool in caring for the complex maternal medical patient. Often the ultrasound tells about fetal health but it can also be a window into maternal health.

\section{Fetal Growth Abnormalities}

Fetal growth abnormalities on either end of the spectrum may be a manifestation of maternal disease. Therefore, while maternal disease warrants ultrasound screening for fetal growth abnormalities, conversely, fetal growth abnormalities should trigger consideration for undiagnosed maternal disease. Maternal medical conditions associated with fetal growth restriction include pregestational diabetes, renal insufficiency, autoimmune

\footnotetext{
${ }^{1}$ Instructor, ${ }^{2}$ Associate Professor

1,2Department of Obstetrics and Gynecology and Women's Health, John A Burns School of Medicine, University of Hawaii Honolulu, Hawaii, USA

Corresponding Author: Kelly Yamasato, Instructor, Department of Obstetrics and Gynecology and Women's Health, John A Burns School of Medicine, University of Hawaii, Honolulu Hawaii, USA, e-mail: kyamasat@hawaii.edu
}

disease, cyanotic cardiac disease, hypertension, and substance use (e.g. tobacco, narcotics and cocaine). ${ }^{1}$ Fetal growth restriction secondary to teratogenic maternal medications is another way in which growth abnormalities may be manifestation of maternal health.

There is no universal consensus on the definition of fetal growth restriction. The American College of Obstetricians and Gynecologists considers fetal growth restriction to be estimated fetal weight less than the 10th percentile for gestational age. ${ }^{2}$ However, the prospective observational trial to optimize pediatric health in intrauterine growth restriction (IUGR) (PORTO) study found that adverse outcomes were not consistently associated with fetal growth restriction until the estimated fetal weight dropped below the 3rd percentile. ${ }^{3}$

Excessive fetal growth may be described as large for gestational age (birthweight $>$ 90th percentile for gestational age) or macrosomia (fetal weight exceeding 4000 or $4500 \mathrm{gm}) .{ }^{4,5}$ In addition to pregestational and gestational diabetes, maternal gestational weight gain and obesity are also risk factors for macrosomia. ${ }^{6-8}$

Fetal growth abnormalities are also predictive of future maternal health. ${ }^{6,9,10}$ Increased mortality from coronary heart disease has been associated with mothers with a history of either low or high birthweight neonates compared to the reference offspring weighing 2500 to $3999 \mathrm{gm} .{ }^{1}$ The association persists after adjustment for pre-eclampsia, diabetes, and socioeconomic factors. ${ }^{6,10}$ Genotypes linked to both abnormal fetal growth and cardiac mortality may be involved. Pathologic systemic maternal inflammation leading to poor uteroplacental perfusion is another possible etiology of both fetal growth restriction and future maternal disease. ${ }^{11}$ Another potential mechanism is that women with marginal cardiac reserve may be unable to hemodynamically compensate for placental pathology, such as deficient placental growth factor and angiogenesis. ${ }^{9}$ Thus, the resultant fetal underperfusion and growth restriction may be a symptom of otherwise subclinical maternal cardiac disease. Similarly, women with subclinical insulin resistance may be more prone to excessive fetal growth as well as metabolic disease later in life. ${ }^{1}$

\section{Embryopathies}

Major congenital malformations are found in approximately 3 to $4 \%$ of livebirths. ${ }^{12}$ Nearly half of congenital 
malformations are of unknown etiology, and a large proportion of the remaining cases are multifactorial or attributable to genetic abnormalities. However, approximately 4 to $6 \%$ of malformations in fetuses over 20 weeks gestational age are secondary to teratogenic exposure, ${ }^{3}$ and it is in these cases that maternal health may be a factor.

It is estimated that 60 million reproductive age women worldwide are affected by diabetes mellitus, ${ }^{13}$ and rates of maternal pregestational diabetes are increasing. ${ }^{14}$ Pregestational diabetes is associated with a malformation rate approaching 9 to $10 \%{ }^{15,16}$ Multiple malformations are also increased in this population. ${ }^{15,17}$ Women with gestational diabetes may have a slightly higher risk of congenital malformations as well. ${ }^{18}$ Malformations associated with pregestational diabetes are listed in Table 1.

The risk for congenital malformations in diabetes is related to the degree of maternal hyperglycemia. Schaefer-Graf et al found initial fasting glucose levels of $166 \mathrm{mg} / \mathrm{dl}$ in pregnancies affected by major anomalies in multiple organ systems, $141 \mathrm{mg} / \mathrm{dl}$ if one organ system was affected, and $115 \mathrm{mg} / \mathrm{dl}$ without major anomalies. ${ }^{18}$ First trimester hemoglobin A1c values exceeding 9 to $10 \%$ have also been shown to predict malformations. ${ }^{16,19}$ It is hypothesized that at a cellular level maternal diabetes may increase oxidative and nitrosative stresses that lead to abnormal cell signaling, gene dysregulation, and apoptosis. ${ }^{13}$

Polyhydramnios is another common ultrasound finding in pregnancies complicated by maternal diabetes, and diabetes is associated with up to $25 \%$ of cases of polyhydramnios. ${ }^{20}$ Definitions of polyhydramnios vary, but the most commonly used criteria are a maximum vertical pocket $>8 \mathrm{~cm}$ or amniotic fluid index $>24 \mathrm{~cm}$. The mechanism behind polyhydramnios and diabetes is thought to involve fetal hyperglycemia that leads to an osmotic diuresis with fetal polyuria. Another potential
Table 1: Fetal malformations associated with maternal pregestational diabetes

Cardiovascular defects

- Atrial and ventricular septal defects

- Transposition of the great vessels

- Aortic coarctation

- Tetralogy of fallot

- Total anomalous pulmonary venous return

- Left and right ventricular outflow tract obstructions

Cranial malformations

- Craniorachischisis

- Hydrocephaly

Esophageal atresia

Intestinal atresia

- Duodenal atresia

- Anorectal atresia

Orofacial clefts (Fig. 1)

Hypospadias

Spine malformations

- Caudal regression syndrome/sacral agenesis

Limb reduction defects

Polydactyly

Neural tube defects

- Anencephaly

Renal anomalies

- Renal agenesis

- Ureteral duplication

Anotia/microtia

Aberg A, et al. Congenital malformations among infants whose mothers had gestational diabetes or pre-existing diabetes. Early Hum Dev 2001;61:85-95.

Garner P. Type I diabetes mellitus and pregnancy. Lancet. 1995;346:157-161

Correa et al. Diabetes mellitus and birth defects. Am J Obstet Gynecol 2008;199:237.e1-9.

mechanism is the development of macrosomia with increased fetal blood volume, and therefore, glomerular filtration rate. ${ }^{20,21}$ Concordant to these theories, increasing degrees of maternal hyperglycemia correlate with increasing amniotic fluid volume. ${ }^{22}$ Therefore, a new and otherwise unexplained finding of polyhydramnios should prompt consideration of maternal glucose status.
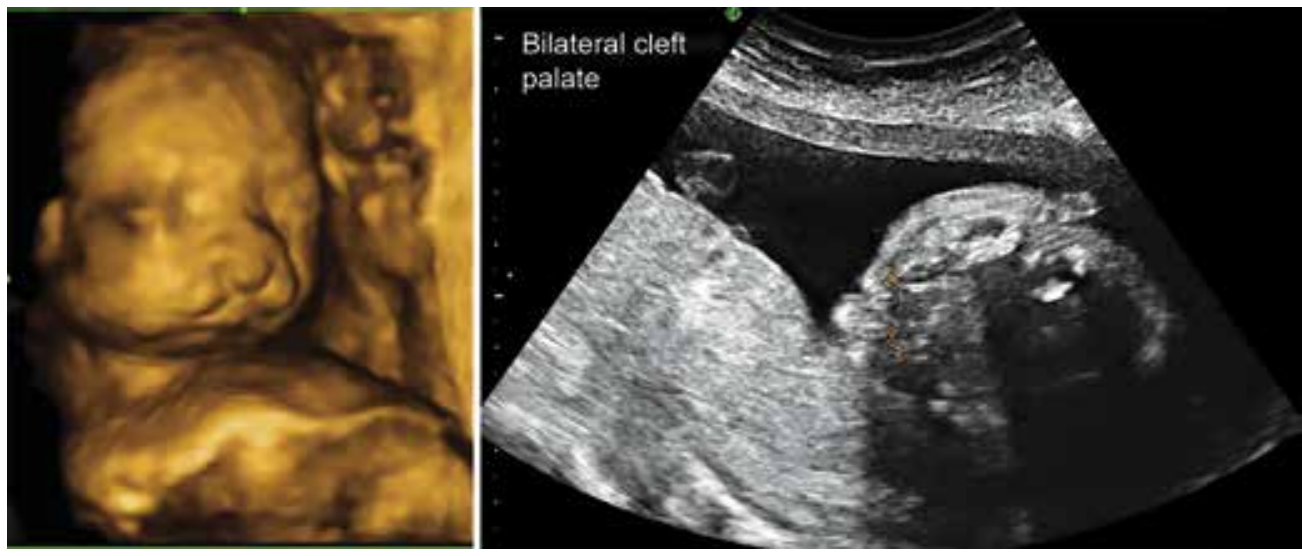

Fig. 1: Bilateral cleft lip and palate at 24 weeks gestation in a patient with pregestational type 2 diabetes mellitus. Her hemoglobin A1c was $9.9 \mathrm{mg} / \mathrm{dl}$ at 20 weeks gestation 
Teratogens are another means through which maternal health impacts ultrasonographic fetal findings. A multinational study found that over $80 \%$ of women reported using at least one medication during pregnancy. Seventeen percent of women used medication for chronic medical conditions. ${ }^{23}$ Some teratogenic medications prescribed for chronic maternal disease include angiotensinconverting enzyme inhibitors, lithium, warfarin, and carbamazepine. Ultrasound findings for selected medications are described in Table 2.

Alcohol abuse and dependence is another maternal condition that may manifest on obstetric ultrasound. Maternal alcohol use is the leading preventable cause of birth defects and developmental disabilities, and based on self-report, $7.6 \%$ of pregnant women use alcohol. ${ }^{24}$ While the findings of fetal alcohol syndrome exhibit a dose-response relationship, there is no known safe lower limit for alcohol consumption during pregnancy. Fetal alcohol syndrome findings have been noted in patients consuming less than one drink per day and in all trimesters of exposure. ${ }^{25}$ While there are no standard criteria for the prenatal diagnosis of fetal alcohol syndrome, prenatal ultrasound findings may include microcephaly, hypertelorism, cardiac anomalies (e.g. atrial and ventricular septal defects),

Table 2: Ultrasound findings in selected teratogenic medications

\begin{tabular}{ll}
\hline Medication & Prenatal ultrasound findings \\
\hline Hydantoin & Hypertelorism \\
& Orofacial clefts \\
Valproic acid & Neurdiac defects \\
& lumbar meningomyelocele \\
& Cardiac defects \\
& Orofacial clefts \\
& Hypospadias \\
& Metopic craniosynostosis \\
& Limb defects, especially radial aplasia \\
& Nasal hypoplasia \\
Warfarin & Stippled epiphyses \\
& Limb hypoplasia, primarily distal digits \\
& Growth restriction \\
& Cardiac defects \\
& Hydrocephalus \\
Isotretinoin & Microcephaly \\
& Microtia/anotia \\
& Micrognathia \\
& Cleft palate \\
& Conotruncal heart defects \\
& Limb abnormalities \\
Spontaneous abortion \\
Ebstein's anomaly (apical \\
displacement of the tricuspid valve) \\
Other cardiac defects
\end{tabular}

Reprotox System (database online). Greenwood Village, CO: Truven Health Analytics, Inc. Updated periodically. Accessed August 1, 2015. renal anomalies, micrognathia, and a single umbilical artery. Fetal growth restriction, if present, is often early onset and symmetrical. ${ }^{26}$ Other findings, such as frontal lobe/transcerebellar ratio, caval-calvarial distance, frontothalamic measurements, and orbital diameter have been evaluated as potential markers for this condition. ${ }^{27}$

Maternal infection with vertical transmission to the fetus is another etiology of congenital malformations. Findings from a selection of these infections are described in Table 3. Cytomegalovirus is the most common congenital infection, affecting up to $2.2 \%$ of neonates. The risk of fetal transmission with primary maternal infection is 30 to $40 \%$ with the greatest risk in the third trimester of pregnancy. Amniotic fluid sampling can detect cytomegalovirus by culture or polymerase chain reaction (PCR), with sensitivities of 70 to $80 \%$ and 78 to $98 \%$ respectively.

Fetal transmission in maternal parvovirus infection approximates 17 to $33 \%$. The most severe effects generally occur with infection prior to 20 weeks gestation. Maternal serologic screening should be performed after possible exposure and amniotic fluid PCR can confirm

Table 3: Prenatal ultrasound findings in fetal infection

\begin{tabular}{|c|c|}
\hline Infection & Prenatal ultrasound findings \\
\hline Cytomegalovirus & $\begin{array}{l}\text { Abdominal and hepatic calcifications } \\
\text { Hepatosplenomegaly } \\
\text { Echogenic bowel } \\
\text { Echogenic kidneys } \\
\text { Ascites } \\
\text { Cerebral ventriculomegaly } \\
\text { Intracranial calcifications } \\
\text { Microcephaly } \\
\text { Hydrops fetalis } \\
\text { Growth restriction }\end{array}$ \\
\hline Parvovirus B19 & $\begin{array}{l}\text { Ascites } \\
\text { Placentomegaly } \\
\text { Cardiomegaly } \\
\text { Hydrops fetalis } \\
\text { Growth restriction } \\
\text { Elevated middle cerebral artery peak } \\
\text { systolic velocity }\end{array}$ \\
\hline Varicella zoster & $\begin{array}{l}\text { Hydrops } \\
\text { Hyperechogenic foci in the liver and } \\
\text { bowel } \\
\text { Cardiac malformations } \\
\text { Limb deformities } \\
\text { Microcephaly } \\
\text { Growth restriction }\end{array}$ \\
\hline Toxoplasmosis & $\begin{array}{l}\text { Ventriculomegaly } \\
\text { Intracranial calcifications } \\
\text { Microcephaly } \\
\text { Ascites } \\
\text { Hepatosplenomegaly } \\
\text { Growth restriction }\end{array}$ \\
\hline
\end{tabular}

American College of Obstetricians and Gynecologists. Cytomegalovirus, parvovirus B19, varicella zoster, and toxoplasmosis in pregnancy. Practice Bulletin No 151. Obstet Gynecol 2015;125:1510-1525. 
the diagnosis with high sensitivity. If acute infection is diagnosed by either serologies or amniotic fluid PCR, ultrasounds every 1 to 2 weeks for 8 to 12 weeks after exposure to monitor for fetal anemia.

Maternal varicella zoster infection may cause congenital varicella syndrome in 0.4 to $2 \%$ of pregnancies, primarily when infection occurs in the first or second trimesters. Maternal infection is often clinically diagnosed, though fluid from the vesicular lesions can be evaluated by PCR to confirm the diagnosis.

Toxoplasmosis is a parasite that can be contracted from undercooked meat or contact with infected cats or soil. Like cytomegalovirus, vertical transmission can occur in all trimesters, but is most likely to occur in the third trimester. Serologies are often inaccurate, and laboratories specialized in the performance of these tests are recommended for suspected infection. Polymerase chain reaction of the amniotic fluid has relatively high sensitivity and specificity. ${ }^{28}$

\section{Congenital Heart Block}

Congenital heart block affects 1 in 20,000 live births. ${ }^{29}$ Half are associated with congenital cardiac malformations, such as left atrial isomerism or congenitally corrected transposition of the great arteries. ${ }^{30}$

The other half of cases of congenital heart block are attributed to anti-Ro/SSA and anti-La/SSB antibodies in the setting of maternal autoimmune disease, generally Sjogren syndrome or systemic lupus erythematosus (SLE). ${ }^{31}$ Conversely, having anti-Ro/SSA or anti-La/ SSB without a prior affected fetus carries a $2 \%$ risk of developing fetal heart block. ${ }^{22,32,33}$ However, in the setting of a prior affected pregnancy the recurrence rate reaches 15 to $18 \% .^{29,34}$ It is believed that the antibodies bind to these antigens in the fetal myocardium, resulting in inflammation and fibrosis of the myocardium and conduction system, particularly the atrioventricular (AV) node. ${ }^{35}$

Heart block is described as 1st, 2nd, or 3rd degree with increasing order of severity. First degree heart block manifests as a prolonged PR interval (> $150 \mathrm{~ms}$ ). Second degree heart block is subclassified into type I (aka Wenckebach heart block) in which a progressively prolonged PR interval precedes a blocked P wave, and type II in which blocked P waves are not preceded by PR prolongation (Fig. 2). Third degree heart block is characterized by completely independent atrial and ventricular contractions in the absence of any conduction through the AV node. While rare, other conduction abnormalities that have been reported in association with anti-SSA/Ro antibodies include prolonged corrected QT interval, sinus bradycardia, cardiomyopathy, and endocardial fibroelastosis. ${ }^{31}$

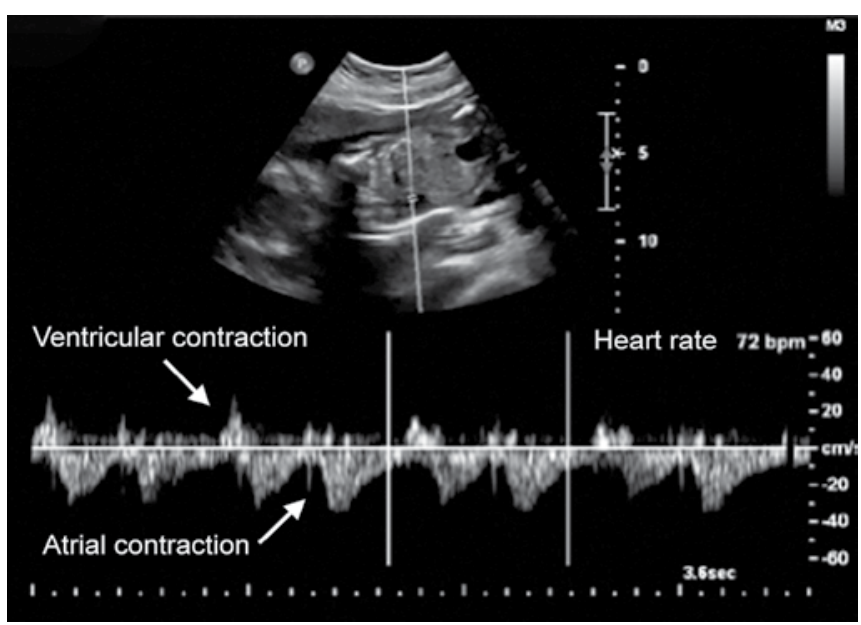

Fig. 2: Pulse wave Doppler demonstrating heart block with a 2:1 conduction pattern with ventricular heart rate of 72 beats per minute

Pregnancies complicated by anti-SSA/Ro and/or antiSSB/La antibodies warrant an initial cardiac evaluation at 16 to 18 weeks for fetal heart block. While some suggest serial echocardiograms every 1 to 2 weeks starting from 16 to 18 weeks gestation to evaluate for the development of heart block, ${ }^{22,29}$ there are no formal guidelines on in utero monitoring.

Congenital heart block may be the first manifestation of maternal autoimmune disease. Thus, this finding in a previously undiagnosed patient should prompt an evaluation of anti-Ro/SSA and anti-La/SSB antibodies. Patients with SLE and Sjogren syndrome in pregnancy require careful ultrasound surveillance through their antepartum course. In addition to the above mentioned cardiac evaluation in the presence of anti-SSA/Ro and/ or anti-SSB/La antibodies, first trimester ultrasound is recommended to confirm fetal viability as these patients are at increased risk for spontaneous abortion. In the third trimester, serial ultrasound evaluation for growth and amniotic fluid is indicated.

\section{REFERENCES}

1. Friedlander Y, Paltiel O, Manor O, Deutsch L, Yanetz R, Calderon-Margalit $\mathrm{R}$, et al. Birthweight of offspring and mortality of parents: The Jerusalem perinatal study cohort. Ann Epidemiol 2007;17(11):914-922.

2. American College of Obstetricians and Gynecologists. Fetal growth restriction. Practice Bulletin No. 134. Obstet Gynecol 2013;121(5):1122-1133.

3. Unterscheider J, Daly S, Geary MP, Kennelly MM, McAuliffe FM, O'Donoghue $\mathrm{K}$, et al. Optimizing the definition of intrauterine growth restriction: the multicenter prospective PORTO study. Am J Obstet Gynecol 2013;208(4):290.e1-6.

4. American College of Obstetricians and Gynecologists. Fetal Macrosomia. Obstet Gynecol 2000;96(5).

5. Langer O. Fetal macrosomia: etiologic factors. Clin Obset Gynecol 2000;43(2):283-297.

6. Bonamy AE, Parikh N, Cnattingius S, Ludvigsson JF, Ingelsson E. Birth characteristics and subsequent risks of 
maternal cardiovascular disease: effects of gestational age and fetal growth. Circulation 2011;124:2839-2846.

7. Crane JM, White J, Murphy P, Burrage L, Hutchens D. The effect of gestational weight gain by body mass index on maternal and neonatal outcomes. J Obstet Gynaecol Can 2009;31(1):28-35.

8. Jolly MC, Sebire NJ, Harris JP, Regal L, Robinson S. Risk factors for macrosomia and its clinical consequences: a study of 350,311 pregnancies. Eur J Obstet Gynecol and Repro Biol 2003;111(1):9-14.

9. Bohrer J, Ehrenthal DB. Other adverse pregnancy outcomes and future chronic disease. Semin Perinatol 2015;39(4):259-263.

10. Bukowski R, Davis KE, Wilson PWF. Delivery of a small for gestational age infant and greater maternal risk of ischemic heart disease. PLoS ONE 2012;7(3):e33047.

11. Cotechini $\mathrm{T}$, Graham $\mathrm{CH}$. Aberrant maternal inflammation as a cause of pregnancy complications: a potential therapeutic target? Placenta 2015;36(8):960-966.

12. Centers for Disease Control and Prevention. Update on overall prevalence of major birth defects-Atlanta, Georgia, 1978-2005. MMWR Morb Mortal Wkly Rep 2008;57(1):1-5.

13. Gabbay-Benziv R, Reece EA, Wang F, Yang P. Birth defects in pregestational diabetes: Defect range, glycemic threshold and pathogenesis. World J Diabetes 2015;6(3):481-488.

14. Lawrene JM, Contreras R, Chen W, Sacks DA. Trends in the prevalence of preexisting diabetes and gestational diabetes mellitus among a racially/ethnically diverse population of pregnant women, 1999-2005. Diabetes Care 2008;31(5):899-904.

15. Aberg A, Westbom L, Kallen B. Congenital malformations among infants whose mothers had gestational diabetes or preexisting diabetes. Early Hum Dev 2001;61(2):85-95.

16. Wender-Ozegowska E, Wroblewska K, Zawiejska A, Pietryga M, Szczaa J, Biczysko R. Threshold values of maternal blood glucose in early diabetic pregnancy-prediction of fetal malformations. Acta Obstet Gynecol Scand 2005;84(1):17-25.

17. Schaefer-Graf UM, Buchanan TA, Xiang A, Songster G, Montoro M, Kjos SL. Patterns of congenital anomalies and relationship to initial maternal fasting glucose levels in pregnancies complicated by type 2 and gestational diabetes. Am J Obstet Gynecol 2000;182(2):313-320.

18. Balsells M, Garcia-Patteson? A, Gich I, Corcoy R. Major congenital malformations in women with gestational diabetes mellitus: a systematic review and meta-analysis. Diabetes Metab Res Rev 2012;28(3):252-257.

19. Hanson U, Persson B, Thunell S. Relationship between haemoglobin A1c in early type 1 (insulin-dependent) diabetic pregnancy and the occurrence of spontaneous abortion and fetal malformation in Sweden. Diabetologia 1990;33(2):100-104.

20. Sandlin AT, Chauhan SP, Magann EF. Clinical relevance of sonographically estimated amniotic fluid volume: Polyhydramnios. J Ultrasound Med 2013;32(5):851-863.

21. Nobile de Santis MS, Radaelli T, Taricco E, Bertini S, Cetin I. Excess of amniotic fluid: pathophysiology, correlated diseases and clinical management. Acta Biomed 2004;75(suppl 1): 53-55.
22. Brucato A, Cimaz R, Caporali R, Ramoni V, Buyon J. Pregnancy outcomes in patients with autoimmune diseases and anti-Ro/SSA antibodies. Clin Rev Allergy Immunol 2011;40(1):27-41.

23. Lupattaelli A, Spigset O, Twigg MJ, Zagorodnikova K, Mardby AC, Moretti ME, et al. Medication use in pregnancy: a cross-sectional, multinational web-based survey. BMJ Open 2014;4(2):e004365.

24. Centers for Disease Control and Prevention. Alcohol use and binge drinking among women of childbearing ageUnited States, 2006-2010. Morb Mortal Wkly Rep 2012;61(28): 534-538.

25. Feldman HS, Jones KL, Lindsay S, Slymen D, KlonoffCohen H, Kao K, et al. Prenatal alcohol exposure patterns and alcohol-related birth defects and growth deficiencies: a prospective study. Alcohol Clin Exp Res 2012;36(4):670-676.

26. Cook JD. Biochemical markers of alcohol use in pregnant women. Clin Biochem 2003;36(1):9-19.

27. Kfir M, Yevtushok L, Onishchenko S, Wertelecki W, Bakhireva L, Chambers CD, et al. Can prenatal ultrasound detect the effects of in-utero alcohol exposure? A pilot study. Ultrasound Obstet Gynecol 2009;33(6):683-689.

28. American College of Obstetricians and Gynecologists. Cytomegalovirus, parvovirus B19, varicella zoster, and toxoplasmosis in pregnancy. Obstet Gynecol 2015;125(6): 1510-1525.

29. Buyon JP, Hiebert R, Copel J, Craft J, Friedman D, Katholi M, et al. Autoimmune-associated congenital heart block: demographics, mortality, morbidity and recurrence rates obtained from a national neonatal lupus registry. J Am Coll Cardiol 1998;31(7):1658-1666.

30. Lai J, Clark TJ, Tan JH, Delaney S, Jolley JA. Ultrasound findings in fetal congenital heart block associated with maternal anti-Ro/SSA and anti-La/SSB antibodies. Ultrasound Q 2015;31(1):34-36.

31. Costedoat-Chalumeau N, Amoura Z, Villain E, Cohen L, Piette JC. Anti-SSA/Ro antibodies and the heart: more than complete congenital heart block? A review of electrocardiographic and myocardial abnormalities and of treatment options. Arthritis Res Ther 2005;7(2):69-73.

32. Ramsey-Goldman R, Hom D, Deng J-S, Ziegler GC, Kahl LE, Steen VD, et al. Anti-SSA antibodies and fetal outcome in maternal systemic lupus erythematosus. Arthritis Rheumatism 1986;29(10):1269-1273.

33. Brucato A, Frassi M, Franceschini F, Cimaz R, Faden D, Pisoni MP, et al. Risk of congenital complete heart block in newborns of mothers with anti-Ro/SSA antibodies detected by counterimmunoelectrophoresis: a prospective study of 100 women. Arthritis Rheum 2001;44(8):1832-1835.

34. Julkunen $\mathrm{H}$, Eronen M. The rate of recurrence of isolated congenital heart block: a population-based study. Arthritis Rheum 2001;44(2):487-488.

35. Llanos C, Friedman DM, Saxena A, Izmirly PM, Tseng CE, Dische $R$, et al. Anatomical and pathological findings in hearts from fetuses and infants with cardiac manifestations of neonatal lupus. Rheumatology 2012;51(6):1086-1092. 